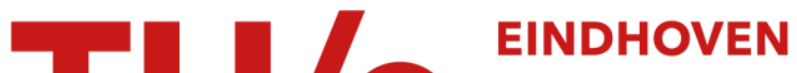 \\ UNIVERSITY OF \\ TECHNOLOGY
}

\section{Human operator research with a simulated distillation process}

\section{Citation for published version (APA):}

Paternotte, P. H., \& Verhagen, L. H. J. M. (1976). Human operator research with a simulated distillation process. (Technische Universiteit Eindhoven. Fac. der Bedrijfskunde. : rapport). Technische Hogeschool Eindhoven.

Document status and date:

Published: 01/01/1976

\section{Document Version:}

Publisher's PDF, also known as Version of Record (includes final page, issue and volume numbers)

\section{Please check the document version of this publication:}

- A submitted manuscript is the version of the article upon submission and before peer-review. There can be important differences between the submitted version and the official published version of record. People interested in the research are advised to contact the author for the final version of the publication, or visit the $\mathrm{DOI}$ to the publisher's website.

- The final author version and the galley proof are versions of the publication after peer review.

- The final published version features the final layout of the paper including the volume, issue and page numbers.

Link to publication

\section{General rights}

Copyright and moral rights for the publications made accessible in the public portal are retained by the authors and/or other copyright owners and it is a condition of accessing publications that users recognise and abide by the legal requirements associated with these rights.

- Users may download and print one copy of any publication from the public portal for the purpose of private study or research.

- You may not further distribute the material or use it for any profit-making activity or commercial gain

- You may freely distribute the URL identifying the publication in the public portal.

If the publication is distributed under the terms of Article 25fa of the Dutch Copyright Act, indicated by the "Taverne" license above, please follow below link for the End User Agreement:

www.tue.nl/taverne

Take down policy

If you believe that this document breaches copyright please contact us at:

openaccess@tue.nl

providing details and we will investigate your claim. 
The object of the research is the task-situation of the human operator in more-or-less automated man-machine systems. More precisely, we are interested in the operator's role in the system: the skills he needs and how to train these, the cognitive processes involved in control and supervisory tasks, the satisfaction operators find in their work and the interaction between the lay-out and design of the technical equipment (especially the interface) and the operator's control behaviour and concrol performance. The aim of this research 
This paper is concerned with the research activities on manmachine systems at the Department of Industrial Engineering at Eindhoven University of Technology. This research is carried out in field situations as well as in the department laboratory by means of a digital simulation of an industrial distillation process.

After a brief survey of research topics, the paper focues first on a detailed description of simulation soft- and hardware as well as of special software for control of exgonomic experiments. In the second part the results of a pilot-study with the simulated process are presented. The aim of this study was to acquire experience with the simulation itself and to investigate the learning behaviour of experienced and non-experienced subjects.

At the same time the effects of speeding up the simulation on control performance and control behaviour were investigated. 
is to provide data in order to optimize future and existing manmachine systems with respect to technical, economic and social criteria.

Field research is done at the chemical industries DSM in the southern part of the Netherlands. The results reported by Kragt and Landeweerd (1975) provided the guidelines for further activities of both continued field research (Paternotte, 1975) and the development of a simulated process in the laboratory (Verhagen, 1975).

The laboratory plant is a copy of an existing distillation column at DSM. This set-up offers fruitful possibilities for implementing and validating experimental results. At the same time it will be possible to answer questions about the real situation that cannot be explored there, due to the uncontrollability of several disturbing sources and the costs involved.

The choice of an industrial distillation plant as a research tool was based upon the following considerations:

It is very difficult to generalize research findings from complex man-machine systems (e.g. electric energy plants, chemical plants, air traffic control systems) to each other. Therefore it seems useful to start research with a widespread industrial process like distillation. Another important consideration was the accessability of the field situation and the cooperation and participation of control engineers and production staff for which DSM offered the best opportunity. Moreover, the set-up of the simulation was largely facilitated by the existence of a static control model of the distillation plant, kindly made available by DSM.

\section{RESEARCH PROJECTS}

At this moment the following research projects are started or in progress (more detailed information can be found in Patemotte, 1976): 
2.1. Intermal Representation. Important aspects of the task of the human operator are of a mental nature. It is assumed that the operator has an internal representation of the process he is controlling. In this research the effect of factors as: intelligence, education and experience on the development of that internal representation are investigated as well as the relation between internal representation, control performance and control behaviour.

The influence of several individual factors on that rolation is studied as well.

2.2. Worksatisfaction of Operators. Subject is the kind of relation between the work-intrinsic factors, traits of the operator and pleasure in performing the job. The aim is to develop a method with which tasks can be constituted, so that these will suit the best to the needs of the operator.

2.3. Information and Performance. The investigation aims to determine relations between nature and quantity of information and control performance.

Alternative ways of information presentation are developed and compared.

Analyses of the visual scanning behaviour will be used to determine the subjective uncertainty of operators in relation to the information presented.

2.4. Humon Reliability. The first aim is the definition of the concept of human error and the estimation of probability of occurence frequencies in real situations. By means of the critical incident technique the factors affecting human reliability will be identified. This concerns both aspects of human operators (e.g. skills, training, personality traits) and technical aspects (e.g. interface design, failure rates, etc.). The next step will be the investigation of these factors in the laboratory in order to quantify their relative importance to human reliability.

2.5. Classification of Tasks and Abilities. Obviously, the benefits of research will be greater if the results can be applied to a diversity of control tasks. We consider a 
classification system as a set of at least nominally ordered categories in terms of which all objects in a defined set can be objectively described.

Different objects can be compared when they are described in terms of the same classification system. To be of use for the mentioned research projects, the classification system must allow the ordering of tasks as well as abilities.

2.6. Expemiment Control. Software is developed for a wide variety of experiments. Special attention is paid to on-?ine communication between the experimenter and the current experiment. The experimenter has the possibilities to monitor, finish, (re)start and freeze an experiment. He can determine beforehand or during an experiment to be provided with information concerning control performance, control behaviour and process state. Off-line techniques are developed and sophisticated for data compression, data handing and data analysis.

\section{OUTLINE OF THIS PAPER}

The foregoing presentation of backgrounds and research projects was meant to present the context in which the following paragraphs must be seen, and to give a wider bearing to the information concerning the man-machine system research projects.

The following sections discuss in more detail two of the before mentioned projects. The primer is a detailed description of the simulation soft- and hardware together with a description of experiment and data handling techniques (see 2.6.). The latter presents the results of a pilot-study with the simulator. This study was part of the project Information and Performance (see 2.3.) and was aimed at assessing data on operator performance, learning effects and effects of increasing simulation speed. 
4.1. The Simulated Process

The process used for research on operator behaviour, is a single distillation column with 63 trays, $34 \mathrm{~m} \mathrm{high}$. The simulation is based on a model of a real column at DSM. It is a part of a plant of four distillation colums. Simulation of the whole plant, in order to build a complete operator task, is a goal for the near future. In the simulated process (see figure 4.1.), a two component fluid (feed) is distilled into a pure top-product (1ight component) and a bottom-product (heavy component). The topproduct has a lower boiling temperature than the bottom-product. The system goal which the operator has to accomplish, sets requirements on the purity of both products, as well as on the energy consumption. The required impurity values are $0.05 \%$ heavy component in the top-product (YD) and $5 \% 1 \mathrm{ight}$ component in the bottom-product $(\mathrm{XB})$. The energy constraint has not yet been used in laboratory experiments. In the present state the operator may use a maximum of 3 degrees of freedom to achieve the system goal. These are the settings of feed-flow, reflux flow and temperature of tray 15. The latter forms a cascade control loop with the setting of the steam flow. In reality the freedom in use of the feed-flow as control variable is restricted or zero (constant feed). The fourth degree of freedom in the simulation is the feedconcentration. This concentration however is constant during an experiment or is changed by the experimenter, resembling the real situation. The four degrees of freedom influence strongly the concentrations and flows of both top and bottom products. The transfers from setpoint to output concentration have tine lags up to 10 minutes and rising times of up to one hour. This implies a very slow responding system, which may take several hours before the change of a setpoint has stopped showing influence at the output. 
Information about the process variables is displayed on a control pane1, see figure 5.3. and sections 5.2.3., 5.2.4.

\subsection{Hardware configuration}

The simulation has been implemented on a DEC PDP $11 / 40$ minicomputer with $32 \mathrm{k}$ core memory under RT 11 Foreground/Background operating system. The current simulation program including experimenter's interaction and CRT usage requires about $10 \mathrm{k}$ of memory.

Figure 4.2. shows a diagram of the configuration (Digital, 1973). The analog and digital input and output registers (16 bit) in the LPS module are used for data transfer to and from the contro1 pane1:

- 8 Analog inputs are used for transfer of setpoint-information from 10-turn potentiometers.

- 1 Digital output register is used to drive 64 output channels. 6 Bits are used for addressing 64 DA-converters, 10 bits contain the output information.

- 1 Digital input register is in use to transfer certain functions which can be set by switches.

- 1 Digital input register will be used for switching manual and automatic control on some contro1 loops.

- 1 Digital output register nay be used to activate alarms. An input-output bus system will be installed to provide more flexible consoles for the operator as well as for the experimenter.

\subsection{The Simuzation Software}

\subsubsection{General Approach of the Simulation}

The simulation model is not a full dynamic representation of the physical distillation process. It consists of a static part which calculates the final $(t=\infty)$ values of the output concentrations as a function of the current setpoint values. In the real plant this program is used as an advisory model for operators. A chain of first order processes with different time lags and rising times simulates dynamic responses. This so-called overall approach for simulation has certain disadvantages compared to tray to tray modelling. It will not simulate all dynamic responses automatically. 
Responses which are not foreseen have to be added separately. For example, the dynamics of the temperature of tray 15 consists of separate, superposed equations for changes in steam flow, feed flow and external reflux. Small disturbances can be put on some variables. Breakdown, starting-up and shutdown routines have to be simulated separately.

The advantage of this approach was that the implementation could be done fast, starting from the existing static mode1. So there was no need to describe complex physical relations in oreat detail.

The model has been validated for a few process conditions with average throughput. A temperature profile, which is frequentiy used by trained operators providing feedforward information, could as yet not be reliably simulated because of a lack of fixation points.

An advantage from the experimenter's point of view is the calculation of static values itself. These have to be known in order to calculate the dynamic responses. Thus at eny during simulation, a prediction is available of the final values of the output concentrations. This can be presented to the experimenter as a help to control the experiment. In a later stage of research, the influence of presenting them to the operator will be investigated. For real-time operation of the column the full set of equations has to be calculated once every 10 seconds. This provides sufficient accuracy with respect to the rising times. Input and output are done 10 times as fast for realistic responses on the control panel. A shorter cycle time speeds the simulation up. The experimenter thus can change the operator's task or even the apparent plant dynamics. Of course this is a very useful feature during model development and program testing. The maximum speed which now can be obtained is about 25 times real-time. 


\subsubsection{Specific Description of Moduzes}

The sequence of the different program modules in the simulation is controlled by a so-called experiment monitor (see figure 4.3.) from which the modules are called as subroutines. A programmable clock controls the timing during dynamic simulation. The cycle time can be changed by changing the contents of the clock buffer from which clock ticks are countoz. Elags can be set on-line to put the simulation in certain modes (see further). Following are descriptions of some of the modul.. tin some, the first few instructions test flags to determine whether or not the full module will be executed.

1 Initialize: initializes the column in a stable process sctte; brings the column in a new stable process state immedictely after setpoint changes; calculates static values of othut. concentrations.

2. Input, output: reads analog inputs from the potmeters anc performs $A D$ conversion; fills the output register wa... output value and address of a DA converter.

3. Display output: controls output to the expormenter"s CRT. A process diagram is generated and current values of variables are displayed (see figure 4.4.).

4. Model: calculates dynamic responses of all variables; contains control algorithms and time lag buffers.

5. Critema: updates on-line the values of a fixed set of performance criteria.

6. Data storage: performs periodic storáge of current process state on disk. The set of variables is fixed. The storage interval may be determined at the start of the experiment.

7. Start, info, exit: perform expeximenter oriented functions for experiment control. These functions will be reviewed in the next section. 


\subsection{Experiment Control}

From the beginning of program development for ergonomic experiments with the process simulator, experiment control has been an integral part of the program set. Special attention has been paid to flexibility of interaction between experimenter and experiment. Continuous monitoring of experiments was necessary in an early stage of experimenting. Many different experimental set-ups had to be tested.

The experimenter is seperated from the subject/operator and has a teletype and CRT for interaction with the computer. He starts an experiment from his teletype and may, on demand, get process information on it. The following software modules perform the experimenter oriented functions.

A. Start: the start procedure asks whether or not data are to be stored during the experiment. If so, a file name is asked and a file is opened on disk. A data-storage ir.erval is determined and a data storage flag set. The simulation speed (relative to real-time) and the duration of the experiment are asked for.

B. Stop: tests the "exit" flag, set by the experimenter or the value of the cycle counter to determine the end of the experiment. The simulation is stopped and performance criteria are printed. The number of stored data is given, the datafile is updated and closed.

C. Interaction: the process state can be viewed on CRT. Additional information can be produced on demand. However, in the near future the experimenter only will have a teletype available for experiment control. It has been decided that this will be a11 of an experimenter's console since hardware requirements could as yet not be formulated.

For full monitoring and control of the experiment, the experimenter has the following facilities. All of them are available via two-character commands. 
- Detection of operator action: information can be given on setpoint changes, the new setpoint value and even on the new predicted values of the output concentrations (effect of operator action).

- Process state information: the most important process variables and performance criteria may be printed at fixed intervals $(100,200,300,600$ secs in real-time mode) or in between on demand.

This feature may be important for experiment logging and filing functions especially since files on disk might be destroyed.

- Temporary stopping the experiment: the process can be brought in a "HALT" state. The model is not run, input and output however are still functioning. In addition the process can be brought in the "INITIALIZE" state, a stable process state based on the current setpoint values. Resetting the performance criteria on-line is possible, as well as restarting the experiment.

- Influencing the process: as said before, breakdowns and major disturbances cannot be simulated at the moment. The experimenter can put small steps upon the feedconcentration, in the future also on steam pressure and outside temperature (influence respectively the heatflow and the coldflow in the heat balance). Information presentation of the output concentration on the control panel can be changed from continuous to intermittent presentation, thus simulating chromatograph output.

More facilities can easily be built in since every option is treated as a subroutine. Core space can be saved when using overlays. In the future a multi-tasking operating system will be used in which several program tasks may work on the same simulation data base. 


\subsection{Data-storage and off-ine Analysis}

\subsubsection{Data Storage and Evaluation}

When a data-file is opened by the experimenter, in fact two files are opened. On one, the raw data of the experiment are stored, one set of variables at a time. On the other file data are rearranged after the experiment in blocks of the same variable. At the beginning of the file, some experiment information is stored in ASCII code, and of course the process state from which the experiment is started. It is also possible to store observations, brought into the computer with a push-button box. For quick analysis after an experiment the rearranged data can be inspected on CRI with help of a software package provided by DEC, called SPARTA. One or two variables can be shown on a graphic display at a time. Mathematical calculations can be performed on buffer contents. They can be added to, subtracted from, multiplied with or divided by other buffers and scalars. Smoothing, autocorrelation, crosscorrelation and power spectrum analysis can be performed.

\subsubsection{Off-line Analysis}

Operator behaviour can be studied by observation as we11 as controlaction analysis. It may thus be possible to determine on which base the operator has taken his decisions.

The action-analysis program is run off-line and makes use of the stored data files. It is written in BASIC, being an easy language for program testing and file handling. Lists can be produced of all control actions taken, split per setpoint. Time of action is recorded and the amplitude calculated. Then the average anplitudes are calculated, the average time between actions and variance therein. An interesting measure is what may be called "amount of action". It multiplies per setpoint, for each action the amplitude and the duration of an action, and sums these products over the run. Comparisons can then be made between runs (Verhagen, 1975). Cross correlation techniques are being developed. 


\subsection{Data Reduetion}

The above mentioned data-storage system requires a considerable amount of disk space. However, six full days of experimenting can be stored on one disk. The minimum amount of data needed to replay an experiment consists of starting values plus all actions taken by both operator and experimenter. This reduces the storage room to at least onetenth of the original file.

\subsection{Buture Developments}

Some plans for the near future have already been mentioned. First of all will be the introduction of a multi-tasking operating system in which functions as model, input/output and interaction can be treated as separate tasks. Tasks of lower priority such as information to the experimenter will not interfere any more with tasks of higher priority such as calculation of model equations. A more flexible information presentation to both experimenter and operator may be developed by using CRT and lightpen.

Simulation of the full plant with four distillation columns and storage tanks will be achieved in the next few years.

\section{A PIIOT STUDY WITH THE SIMULATOR}

\subsection{Introduction}

Before initiating research into relations between information presentation, control behaviour and control performance, it was necessary to gain some knowledge about learning as quantified by the improvemen $t$ of control performance due to achieving control skill by continued performance of a control task.

A first step to this is to repeat a standard experiment several times in order to measure the change and rate-of-change of the control performance.

Assuning that there will be no fatigueing effects and that motivation will remain constant, this procedure shall be continued until a stable learning platform is reached. For this purpose the pilot 
study was primarily intended.

At the same time it is important to asses whether control skill is a general skill with respect to a variety of control tasks because otherwise each new experimental control task (or even a slightly modified one) would require a new learning process. This question can at least partially be answered by using subjects with different amounts of previous control task experience.

Another important question is whether the increase of simulation speed will allow less time consuming research, while maintaining the same validity of research findings as compared with real-time research. Therefore one has to compare the results of real-time and fast-time experiments with the same control task. Furthermore there were more general questions concerning the reliability of hard- and software. It was also planned to gather data about the impressions of subjects concerning perceived process fidelity, panel lay-out and the experience of working in a laboratory control room.

The next sections deal with the experiment set-up, results and conclusions.

\subsection{Experiment}

\subsubsection{Experiment Design}

Six subjects carried out six trials each. For each trial the control goal was identical. The first four trials were real-time trials, the fifth was speeded up three times and the sixth ten times. Each trial started with the same stable process state, which deviated from the desired state (as derived from the control goal), thus forcing the subjects to exercise controlaction. The desired state was an unstable situation forcing a subject to continue control action, thus avoiding the possibility of a single lucky control setting.

Each trial lasted for two hours, this being about the minimum amount of time to simulate a more-or-less real control task. The six trials followed each other with at the maximum one overnight pause for each subject. It was assumed that the different pause lengthe vetween 
trials would not effect the control performance and control behaviour scores, the more so because the subjects were allowed to use the time-history information of their previous trials on the panel recorders.

The control performance was measured by the weighted sum of integrals of the absolute error scores of the two concentration values (see also section 4.1.$)$.

In formula:

$\mathrm{P}-\mathrm{score}=\int_{0}^{\mathrm{T}}\left|e_{\mathrm{XB}}\right| d t+100 \int_{0}^{\mathrm{T}}\left|e_{Y \mathrm{D}}\right| \mathrm{dt}$ (i.e. a smaller value of $\mathrm{P}$ means

a better control performance.)

The control behaviour was quantified by the following indicators per control variable (adopted from Verhagen, 1975):

- number of control actions;

- average amplitude of control actions;

- average duration of control actions;

- average time interval between control actions.

The first four trials were meant to asses learning effects. Concerning control performance is was hypothesized that the P-score would decrease over the first four trials and eventually stabilize more-or-less, thus providing a base for comparing real-time with fast-time trials in this respect.

Concerning control behaviour it was hypothesized that learning effects would cause a more efficient control behaviour, meaning a decreasing number of control actions, a decreasing average amplitude of control actions, a decreasing average duration of control actions and an increasing average interval between control actions. The fifth and sixth: trial were meant to explore the effects of speeding up the process. The results of previous experiments lead to the assumption that the speed range three to ten might be useful in future experiments.

\subsubsection{Control task}

For the subjects the distillation control task was formulated as follows: "Your mission is to realize the following values of tre tro 
concentration variables:

- bottom product impurity $\mathrm{XB}=5 \%$;

- top product impurity $Y D=0.05 \%$.

These values are the mean scale readings for both variables. Notice that each deviation is an error; if you have realized a desired value you have to keep it there.

Further you have to consider the following:

- deviations below the desired values are equal to deviations above the desired values (e.g. $7 \%$ XB equals $3 \% \mathrm{XB}$ );

- deviations of $X B$ in percents are equal to deviations of YD in hundredth of percents (e.g. $7 \% \mathrm{XB}$ equals $0.03 \% \mathrm{YD}$ ).

This means that deviations of both concentrations are comparable by scale units on a one to one base.

We start the experiment from a state in which both concentrations are deviating from their desired values, so each minute you delay appropriate control action will increase your error score".

\subsubsection{Control Room}

The experiment is carried out in a control room (CR) with the following dimensions: length 5.0 meters, width 3.75 meters and height 2.5 meters (see figure 5.1.).

Its location is adjacent to the computer room and the experimenter's teletype and CRT (see figure 5.2.). The CR is soundproof according to NR-40. Lighting is continuously controllable between 0-1750 lux. The inside climate is controlled with respect to dxy air temperature, relative humidity and ventilation. During the experiment the following conditions are maintained: lighting 1750 lux (colour temperature $\left.4200^{\circ} \mathrm{K}\right)$, dry air temperature $18-20^{\circ} \mathrm{C}$ and relative humidity $50-60 \%$. The experimenter's facilities include an intercom system to communcate with the $C R$ if necessary. The $C R$ has no windows with the exception of two one-way screens which appear to be mirrors from inside. Further equipment of the CR consists of a table, several chairs and a washbowl with towel, etc. 


\subsubsection{Control Pone?}

The control pane1 consists of three rows of instruments mounted in a vertical double 19" rack (see figure 5.3.). The instruments are located between 1.15 and 1.95 meters above the floor. A11 instruments, with the exception of a horizontal scale-type multi-channel recorder, have a vertical scale with a division in hundred units. The corresponding range of the displayed/controlled variable is indicated by a subscript.

Contro1 knobs of $30 \mathrm{~mm}$ diameter are located at the lower right corner of the instruments (see figure 5.4.). These cover the scale in ten revolutions. The lay-out of the control panel was chosen similar (with some small modifications) to that of the real plant. The following variables are displayed (I) means the value of the variable is indicated, (R) means that is recorded as we11):

- temperature and corresponding setpoint of tray $15(R)$;

- steam flow to reboiler $(R)$;

- top pressure (R);

- level of reflux vessel and corresponding setpoint (R);

- feed flow and corresponding setpoint ( $R$ );

- topproduct flow (R);

- bottom product flow (R);

- reflux flow and corresponding setpoint ( $R$ );

- level of bottom vessel and corresponding setpoint (I);

- concentration of top product $Y D(R)$;

- concentration of bottom product XB (R); (These last two variables are displayed together on a two-channel recorder.);

- temperature profile: six temperatures in the column on a multichanne1 recorder;

- temperature of reflux and corresponding setpoint (R). A11 control loops are from the PI-type. From the control loops only the reflux flow and the temperature of tray 15 were used. The other control settings were fixed during the whole experiment. 


\subsubsection{Subjects}

The subjects were six undergraduate students recruited from several departments of the university (industrial, mechanical and chemical engineering) by means of written anouncements on publication boards. Two of them had experience with process control tasks. One had participated in a previous fast-time (10x) experiment (Verhagen, 1975) the other had been a process operator in the petrochemical industry.

The subjects were paid according to the wage-scales for student employees.

\subsubsection{Instruction}

The subjects were informed about the nature of the research project and the meaning of the experiment. A few days before the experiment started, the subjects received a written instruction with the request to study it. This instruction consisted of a short description of the research project, a chapter with a description of theoretical and technical aspects of distillation and, more detailed, a description of the various effects of changing control loop settings. The instruction ended with questions concerning control decisions in various situations. Prior to the experiment these questions were discussed with the experimenter. When the theoretical aspects proved to be no problem any more, the control panel was explained. By means of some standard questions it was assesed whether the subject knew the various instruments and their functions. The next step was to explain the control task as described in section 5.2.2. A number of standard questions were asked to asses whether the control goal was clear to the subject. If so, the simulation was initialized and the experiment started.

After about thirty minutes the simulation was stopped either by the experimenter of by simulation breakdown. This period was considered to be a familiarization period, essential to give the subject the necessary control feeling. In previous experiments it happened that the simulation mode1 limits were exceeded by subjects without any control experience. Afamiliarization period of thirty minutis proved to be veefu? in prowding the minimum amount of cowtcos still necessary to keep the simulation within limits. This condition 
equalled more or less the real situation: the real process has about the same limit values that should not be exceeded in order to maintain production.

\subsection{Results}

\subsubsection{Leaming Effects}

The P-scores of the four real-time trials are plotted in figure 5.5 .

Figure 5.6. shows the average P-scores. As can be seen in the figures, there is a tendency for increasing performance at the first three trials and a slight decrease at the fourth trial. A first step to test the significance of these differences is the application of the Friedman two-way analysis of variance by ranks (Siegel, 1956).

The computed value of $x_{r}^{2}=15 \quad(K=4, N=6)$. The probability of occurence under $H_{0}$ lies between $p=0.01$ and $p=0.001$. With $a$ rejection region of $\alpha=0.01$, it is concluded that there are significant differences of control performance between the four trials.

The next step is to determine between which pairs of trials a difference exists. This is done by means of the sign test (Siege1, 1956), applied to the pairs of interest, being the combinations of trials $1-2,2-3$ and $3-4$. The results are as follows:

- difference between trial pair 1-2 (6+), p = 0.016;

- difference between trial pair $1-3(6+), p=0.016$;

- difference between trial pair 3-4 (2+, 4-), $p=0.34$. With a rejection region $\alpha=0.05$, it can be concluded that there is a learning effect during the first three trials.

The scores concerning the control behaviour were partly destroyed. This was due to two causes: first, there was no control on correct on-line data storage which caused unobserved creation of incomolete data-files. Second, it was found during off-line data-analysis that several files were intermixed, probabiy durs to ari erroneous file zeorganization procedure.

Measures have been taken to prevent these problems by checking 
data-storage on-line and by copying data-files on a separate disk, only accessible for off-line analysis.

The remaining scores concern those of the subjects 2, 3, 4 and 5 . A Friedman test as described before, shows results as presented in table 1 , column $A$.

The conclusion is that there are no differences.

\subsubsection{Effects of Speeding up the Simulation}

The plotted P-scores in figure 5.5. show a high increase for subject 3 and an exceptionally high increase for subject 4 at trial 5 . As subjects 3 and 4 were the most experienced subjects, they were asked immediately after trial six about their experiences with speeded-up simulation.

Their answer was that they realized too late that they had to do with quickened process responses, although this seemed clear to them at the instruction.

Furthermore they told that during trial six they were alert on quick responses and encountered no more problems.

A Friedman $(K=3, N=6)$ test on the P-scores of trials 4,5 and 6 shows no differences between these trials. The $x_{r}^{2}$ value was 0.33 , having a probability of occurrence, under $H_{0}$, of $p=0.956$. This leads to the conclusion that, with respect to control performance, there is no difference between the real-time and the fast-time (up to 10x) control task.

Of course this is partially due to the nature of this non-parametric test, giving equal weight to the differences in subject scores. However, the fact that only two subjects deviated markedly at trial 5, and for special reasons, seems to make the conclusions based on the Friedman test at least plausible.

Concerning the effects of speeding up on control behaviour the following test procedure was applied:

1. A Friedman test on the results of trials 1 to 5 .

2. A sign test on the scores of trials 5 and 6 .

This procedure was intended to explore both the differences between 
real-time and fast-time trials and between the two fast-time trials.

ad.1. The results are shown in table 1 , column $B$. With a rejection region of $\alpha=0.1$, this means that, with respect to both control variables, the total duration and the average duration of control actions decrease with an increase of simulation speed of $3 \mathrm{x}$ compared with real-time. At the same time the number of temperature control actions decreases. ad.2. The results are shown in table 1 , colunn $C$. Widh a rejection region of $\alpha=0.1$, this means that almost the same effects can be observed when increasing simulation speed from $3 \mathrm{x}$ to $10 \mathrm{x}$ as from real-time to $3 \mathrm{x}$. A new effect is the decrease in average interval between control actions. These findings however are not surprising when one realizes that the amount of time available for the fast-time trials decreases in proportion to the increase of simulation speed. 


\subsubsection{Effects of Previous Experience}

By observing the plotted P-seores in figure 5.5. of trials 1 to 4, it $c$ an be seen that the subjects with previous experience do not deviate a great deal from the others. Subject 4, the one with experience in real plants, shows the strongest effects: at the trials 1,2 and 4 he has the lowest P-score although these differences are not large. Subject 3 , the one with simulation experience, even has the highest score at trial 1 but does not differ from the others at trial 2, 3 and 4. As a statistical test of significance of these differences, the Kendall coefficient of concordance, $W$, is computed for the rankings of P-scores of trials 1 to 4 for each subject (Siegel, 1956).

W was found to be .44 with $s=122(N=6, K=4)$.

The critical value at the 0.05 level being $s \geq 143,3$ the conclusion is that no association exists between subjects concerning their control performance. However, as subject 4 accounts for the greater part of the s-value ( 81 out of 122) it seems that experience has some effects in that it leads to a constant position in the lower P-score region. Concerning the control behaviour, the same test (for $\mathrm{K}=4, \mathrm{~N}=4$ ) is applied.

Significant $W$ values are found for the following indicators of control behaviour $(\alpha=0.05)$.

- numver of temperature control actions;

- number of reflux control actions;

- average interval between temperature control actions;

- average interval between reflux control actions;

- average amplitude of reflux control actions.

The first four effects are most likely due to subject 5, having a high score with respect to the number of control actions and a low score with respect to the average interval between control actions. Of course with a high number of control actions the average interval between control actions will be shorter. The only effect probably related to experience has to do with the average 
amplitude of reflux control actions. This is due to subject 4, who has a markedly lower score.

\subsection{4. 'Subjects' Comments}

The general impression of subjects concerning the fidelity of the simulation was that it meeted their expectations as based on their various backgrounds. An exception was made for the readings of the temperature profile recorder. Three subjects said to believe that this instrument did not provide realistic information (this was a correct diagnosis, the temperature profile being a rough approximation requiring improvement, see section 4.3.1.). The control panel was less approved of. Both the panel lay-out and the panel type (requiring a standing position) were critized with respect to various ergonomic criteria. A further remark was that the panel lay-out was less important during fast-time simulation, especially with a speed factor of 10 , because the only readings used in that situation were the control loop variables and the concentration variables.

The control room and control room conditions were generally approved of.

\subsection{Conctusions and discussion}

The results of the pilot-study lead us to the following conclusions: With respect to leaming effects, the hypothesized effects are observed at the control performance scores, but not at the scores of the control behaviour.

With respect to the effect of speeding up the simulation, it is found that the control performance does not change with the speed factors $3 \mathrm{x}$ and $10 \mathrm{x}$. The observed changes at the scores of the control behaviour have only to do with technical aspects of fast-time simulation and are not due to changes in control behaviour itself. However, from the subjects'comments it can be concluded that, with increasing simulation speed, the control strategy changes from feed-forward control to feed-back control. This stresses the need to look for alternative measures of control behaviour e.g. measures of scanning behaviour. With respect to the effects of experience no conclusive results are found. 
Considering these conclusions the following comments can be made: the most important outcome is the observed learning effect as measured by changes in control performance. This outcome indicates the possibility of training unexperienced subjects on the laboratory control task in one day, thus providing a starting point for further, more sophisticated research. Of course there is the possibility that learning is not finished with respect to improvement of control performance, but this can possibly be controlled by intermediate checks with the described standard control task. The effects of speeding up the simulation seems promising with respect to future fast-time simulation research. However, there is a factor involved that has not yet been mentioned. The control task used in this study is adopted from a real situation in which one operator controls eight distillation columns simultaneously. Assuming that this does not present an excessive mental taskload for the operator, it seems reasonable to assume an underloading of the subjects, especially after the learning trials. Increase of simulation speed will probably increase the mental taskload. A cue to this may be that several subjects reported a reduced use of displays with increasing simulation speed. Therefore the fact that control performance does not change may be due to spare mental capacity. In real situations and more extensive laboratory tasks (as planned), this may be different. A tentative conclusion is therefore that speed factors up to $3 x$ may be useful. Another question may arise concerning the application of non-parametric tests. This suggests a mere ordinal measurement of control performance and control behaviour. It can be argued that the p-scores are measured on a ratio-scale. Moreover, the subjects actually had to use a ratio-scale in estimating deviations from their control goal.

The aim of this study, however, was not a precise determination of differences of control performance but was meant to answer questions like: How quick will subjects learn the control task? And what are the effects of speeding up the simulation? Therefore it was decided to start with a rough analysis. In a later stage of the research project the data will be compared more precisely with those of other experiments. 
Kragt, H., Landeweerd, J.A., (1975) Mental Skills in Process Control. In: E. Edwards and F.P. Lees (eds.): The human operator in process control. Taylor and Francis, London. Peripherals and Processor Handbooks PDP 11, 1973. Digital Equipment Corporation, Massachussets. Paternotte, P.H., (1975) The control performance of operators. Paper presented at the Second International Symposium on Man-Machine Systems and Environment, Dubrovnik 16-18 oct., 1975, Yugos lavia.

Paternotte, P.H. (1975) (editor) Short survey of research activities of the Research Group on Man-Machine Systems. Department of Industrial Engineering, Eindhoven University of Technology.

Verhagen, L.H.J.M., (1975) Computer Programs for data management and experiment control with a simulated process. Paper presented to the Second International Symposium on ManMachine Systems and Environment, Dubrovnik 16-18 Oct., 1975, Yugos lavia.

Siege1, S., (1959) Non-parametric statistics for the bellavioral sciences. McGraw-Hil1. 
Table 1. Results of statistical tests on a number of control behaviour indicators. ( $*$ means at least significant at 0.1 level).

\begin{tabular}{|c|c|c|c|c|c|c|}
\hline \multirow[b]{2}{*}{ Indicator of control behaviour } & \multicolumn{2}{|l|}{ A } & \multicolumn{2}{|l|}{$B$} & \multicolumn{2}{|l|}{ C } \\
\hline & $x_{r}^{2}$ & s & $x_{r}^{2}$ & s & $\mathrm{p}$ under & $\mathrm{s}$ \\
\hline Number of temperature control actions & 5.625 & & 10.8 & $*$ & 0.69 & \\
\hline Number of reflux control actions & 3.675 & & 4.15 & & 0.69 & \\
\hline Average amplitude of temperature control actions & 0.825 & & 3.15 & & 0.69 & \\
\hline Average amplitude of reflux control actions & 2.7 & & 3.8 & & 0.31 & \\
\hline Total duration of temperature control & 4.42 & & 8.55 & $*$ & 0.31 & \\
\hline Total duration of reflux control & 2.175 & & 7.85 & $*$ & 0.06 & $*$ \\
\hline Average duration of temp. control actions & 0.075 & & 8.05 & $*$ & 0.06 & $*$ \\
\hline Average duration of reflux control actions & 4.725 & & 11.15 & $*$ & 0.06 & $*$ \\
\hline Average interval between temp. control actions & 1.5 & & 7.4 & & 0.06 & $*$ \\
\hline Average interval between reflux control actions & 0.6 & & 1.4 & & 0.06 & $*$ \\
\hline
\end{tabular}

Column A: Results of Friedman test $(N=4, K=4)$, applied to rankings of trial scores of control behaviour for subsects $(2,3$, $4,5)$, trials (1 to 4).

Column B: Results of Friedman test $(N=4, K=5$, applied to ranking of trial scores of control behaviour for subjects $(2,3$, $4,5)$, trials (1 to 5).

Colum C: Results of sign test applied to trial scores of control behaviour for subjects $(2,3,4,5)$, trials $(5,6)$. 


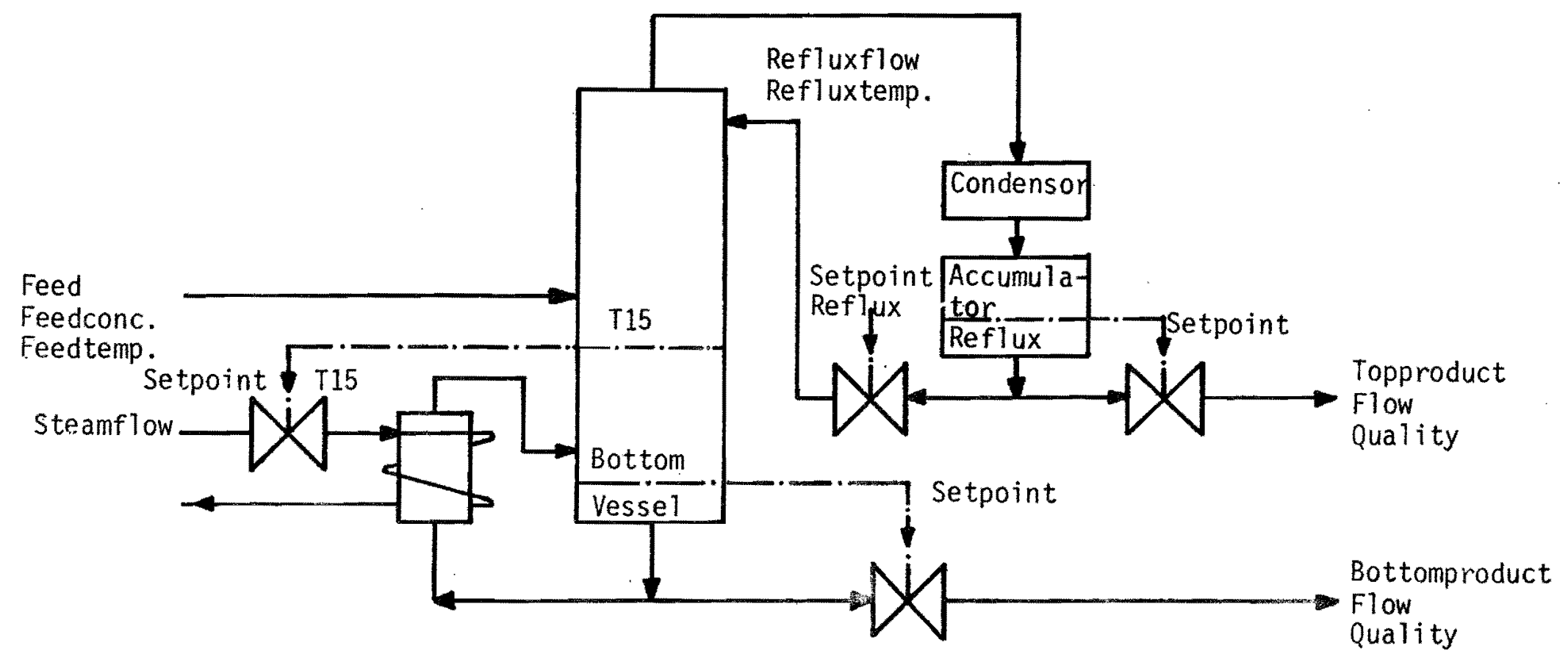

Figure 4.1. Process diagram of distillation column. 


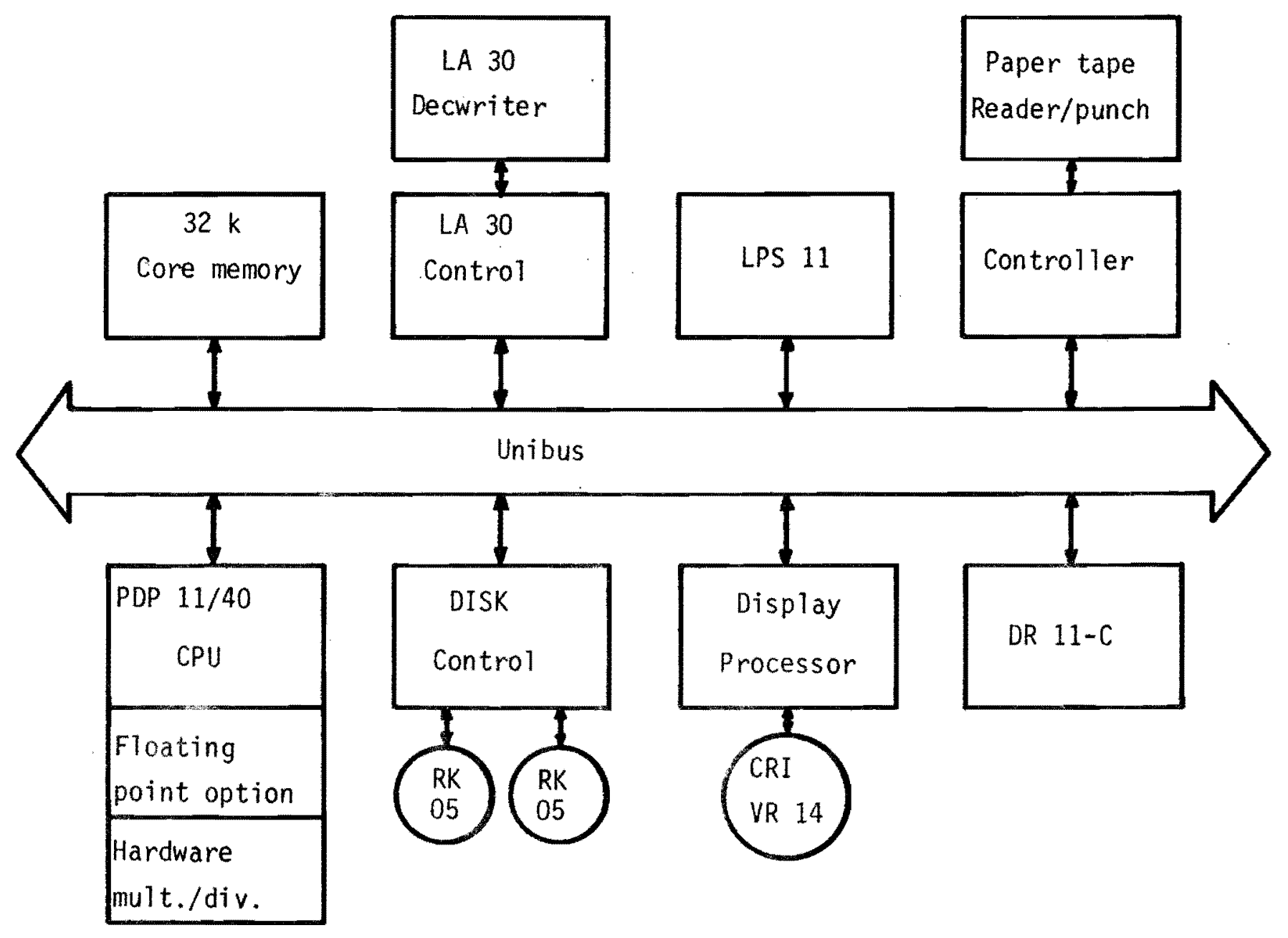

Figure 4.2. Computer configuration. 


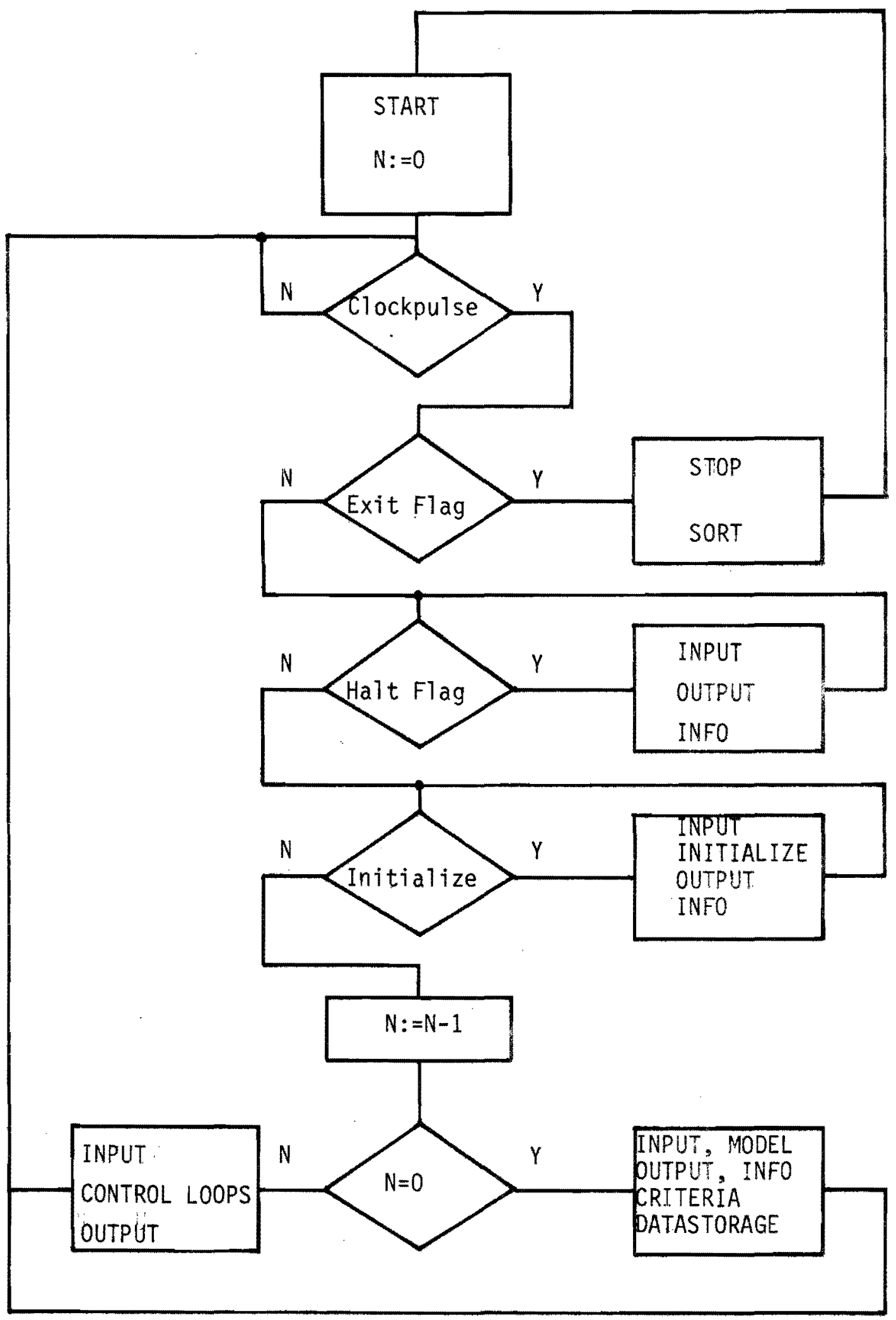

Figure 4.3. Flow chart of experiment monitor. 


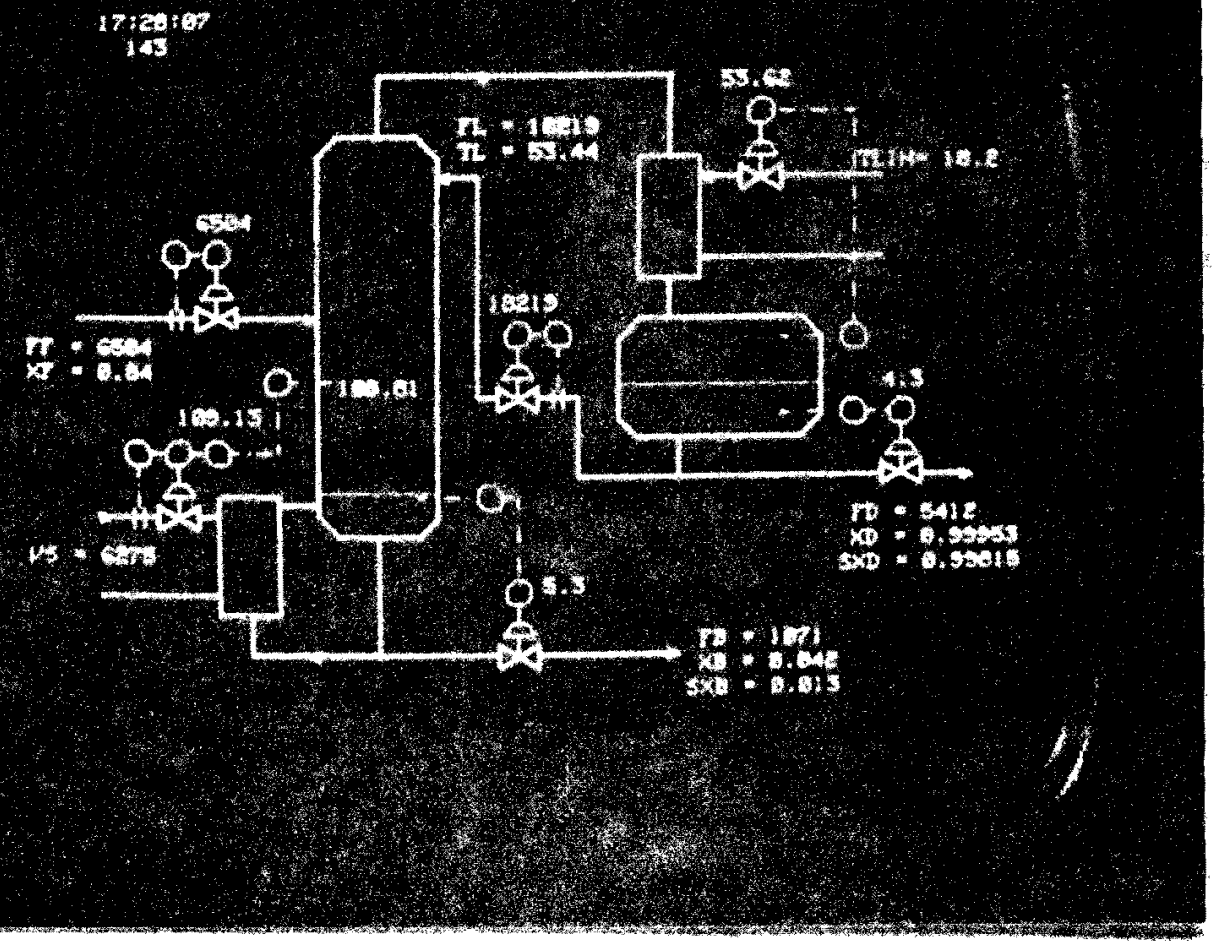

Fig. 4.4. Process Diagram on Experimenter's CRT.

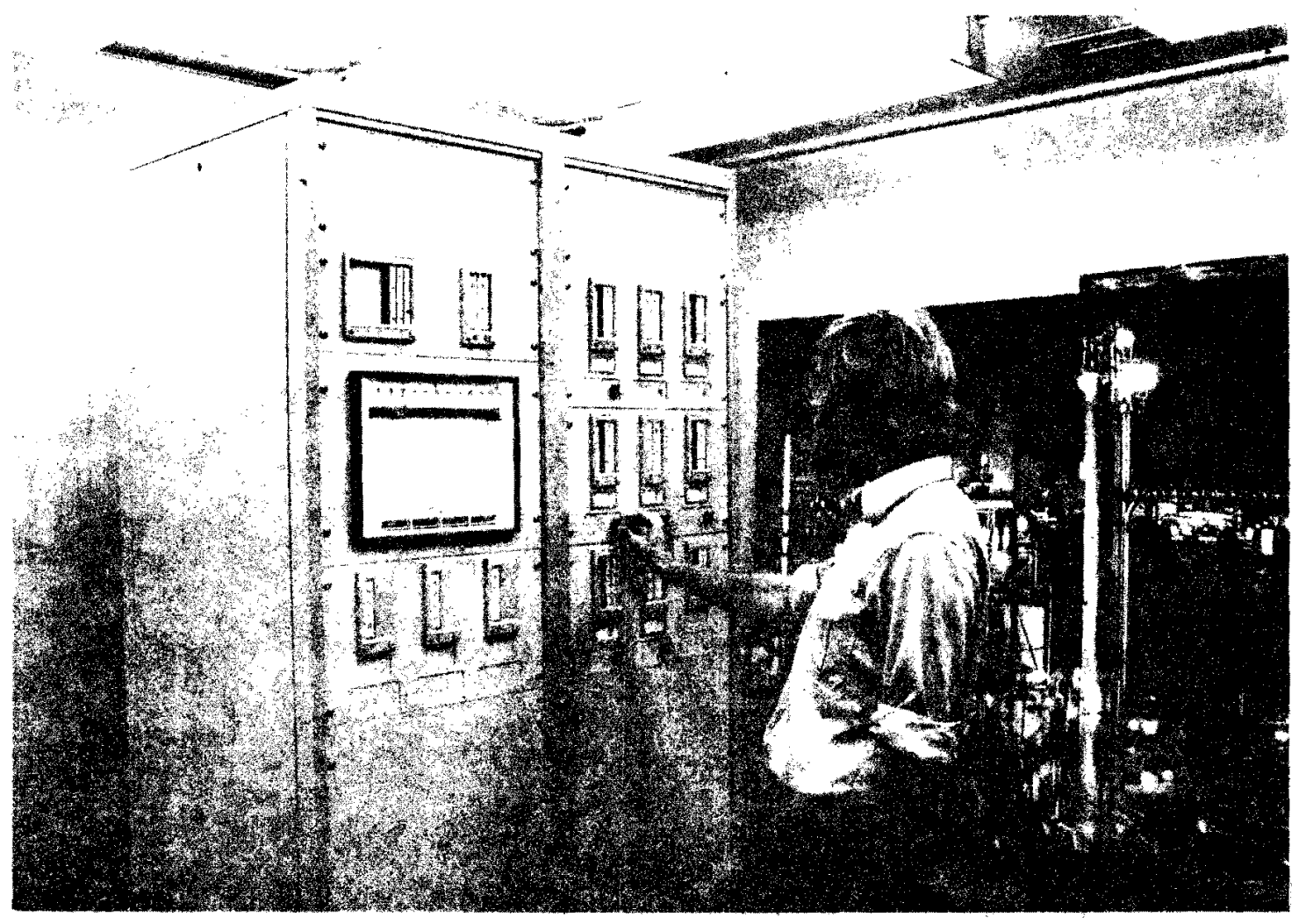

Fig. 5.1. Control Room. 


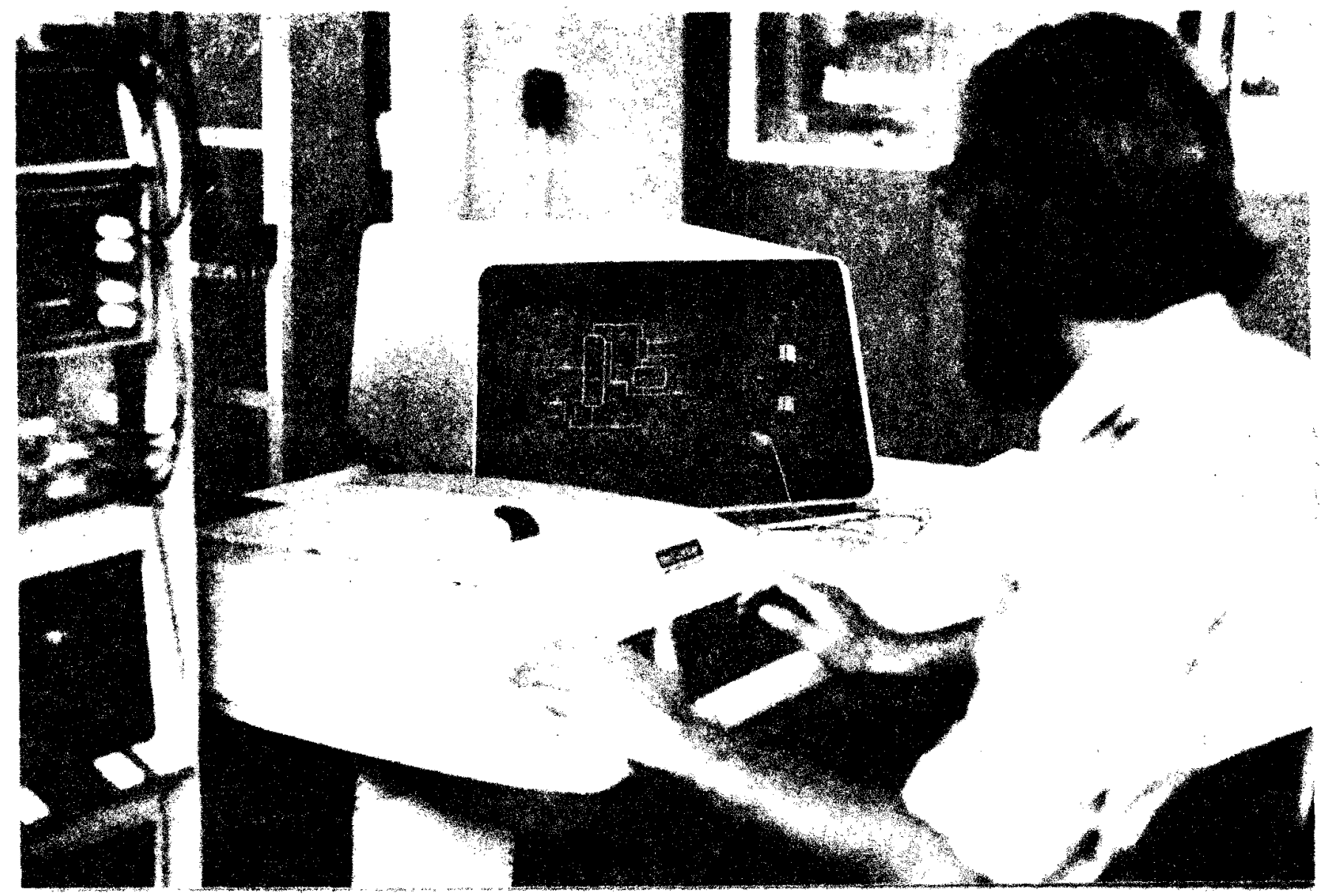

Fig. 5.2. Experimenter.

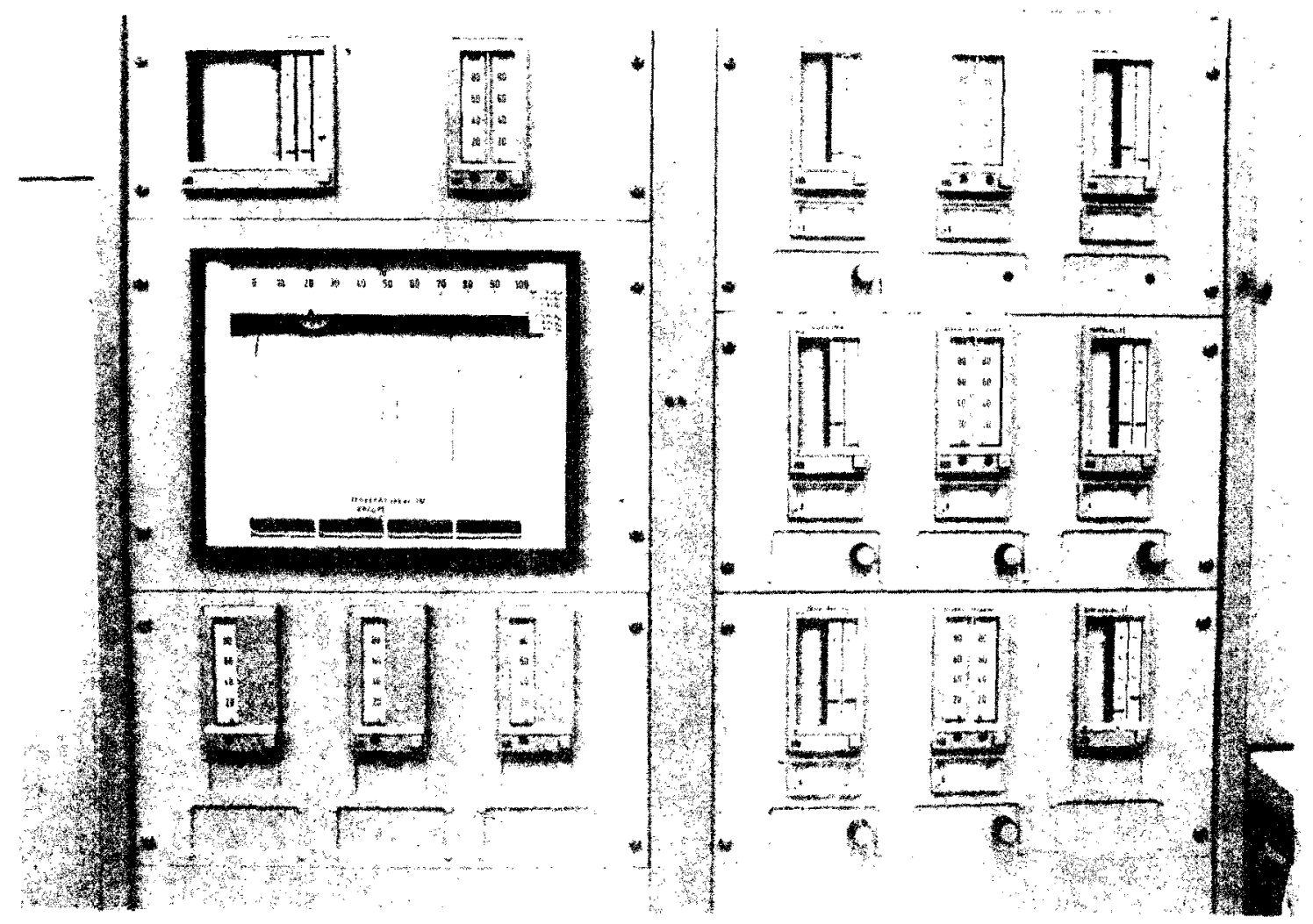




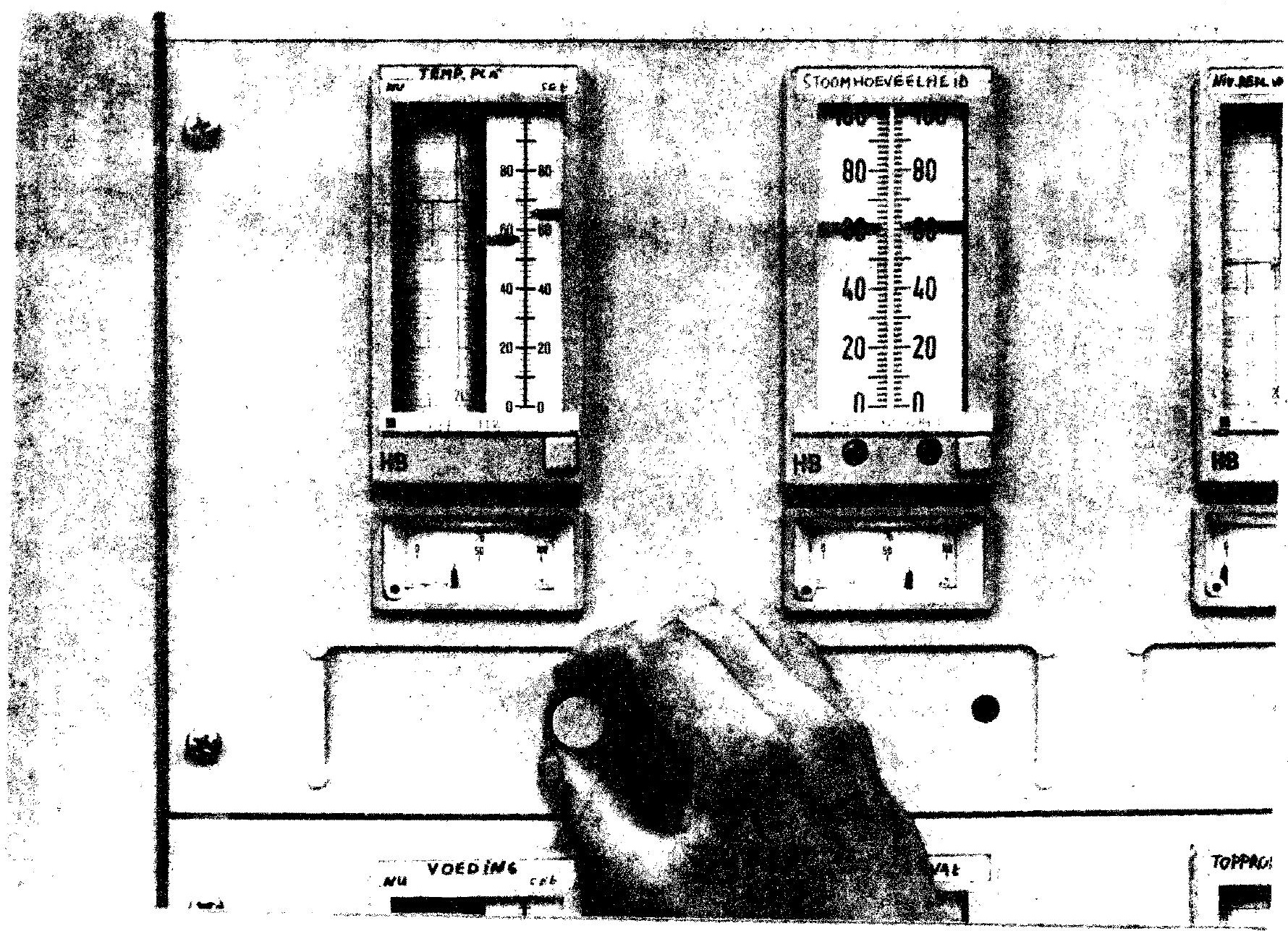

Fig. 5.4. Controller. 


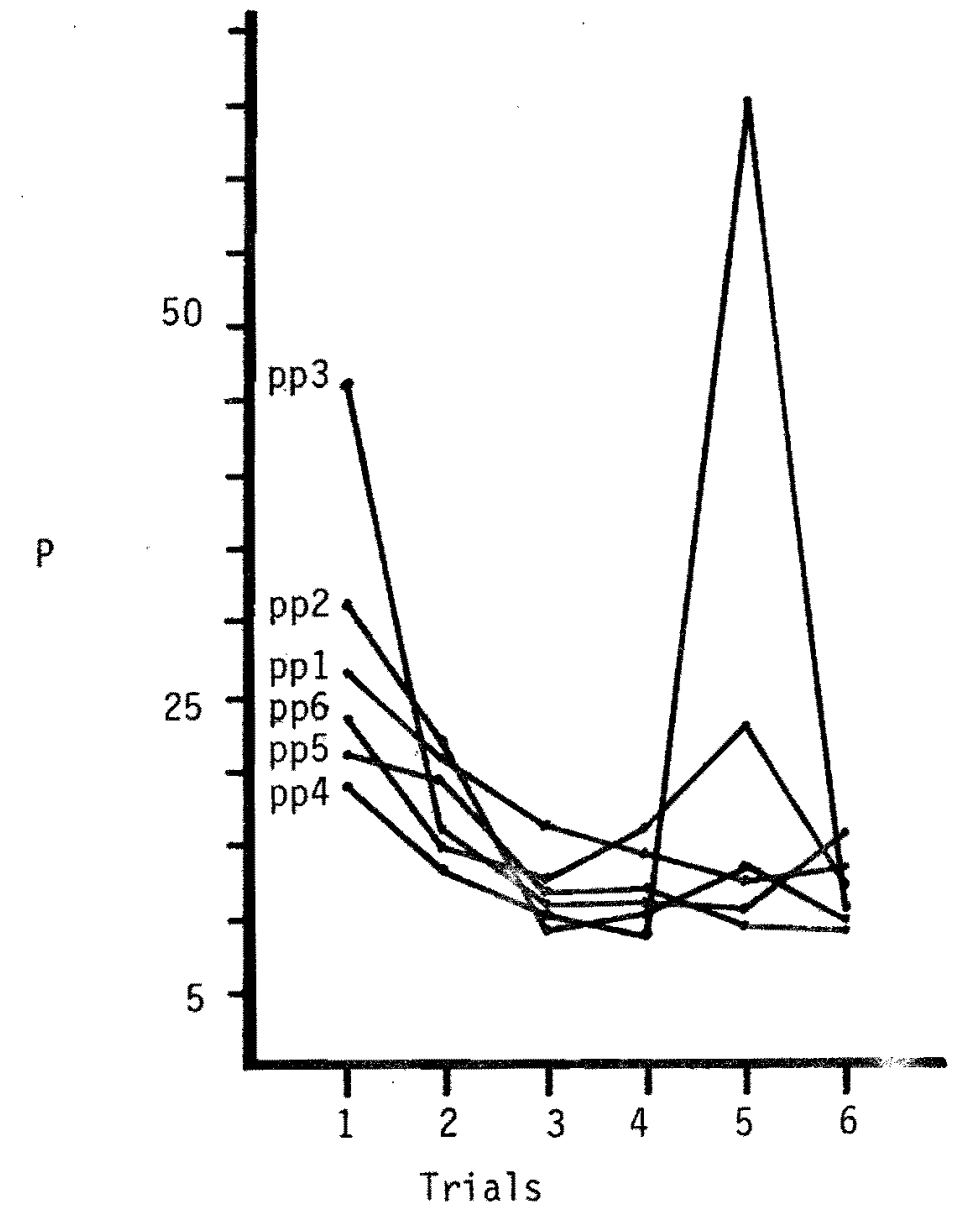

Figure 5.5. Individual P-scores

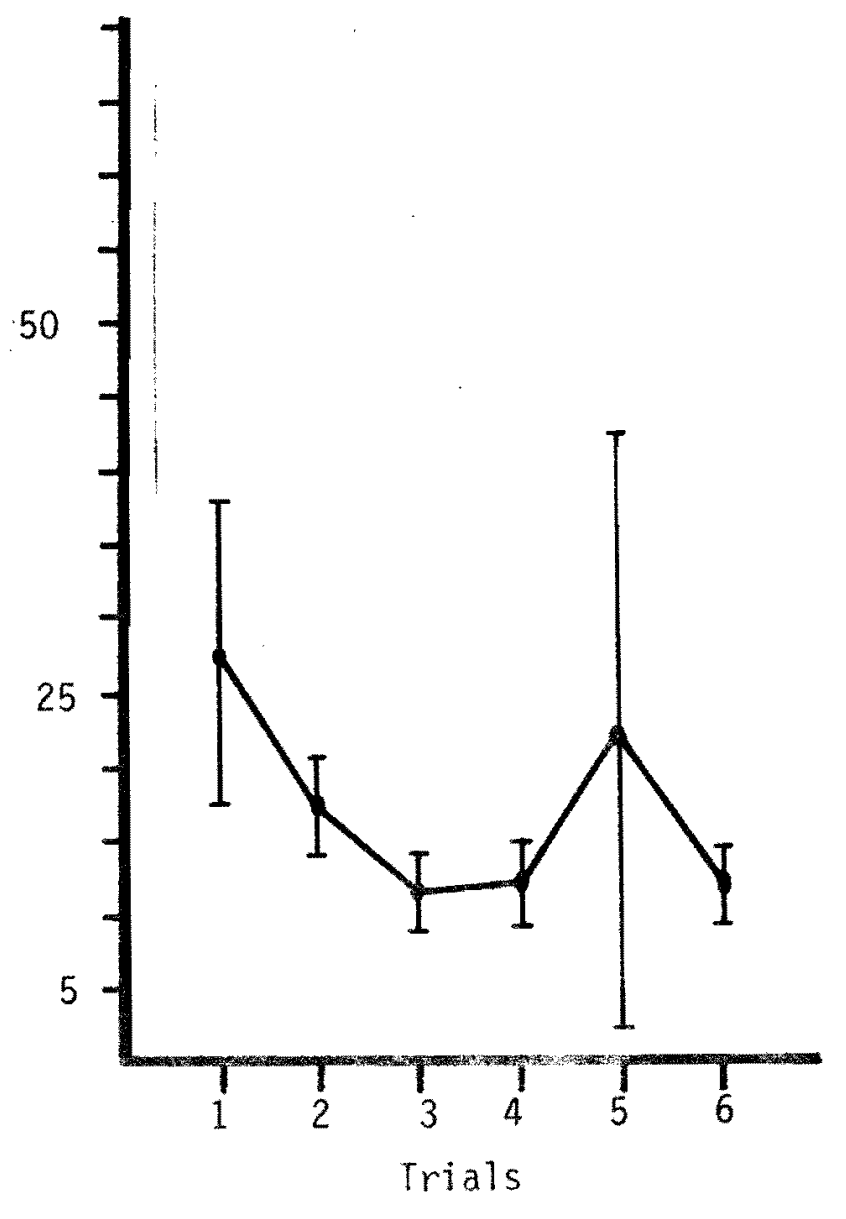

Figure 5.6. Mean and standard deviation of P-scores

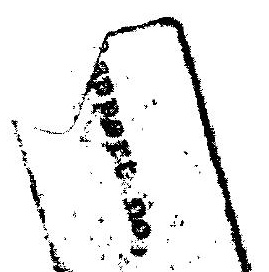

